\title{
BMJ Open Protocol for a systematic review and meta-analysis assessing the effectiveness of deprescribing in falls prevention in older people
}

\author{
Lotta J Seppala (D) , ${ }^{1}$ Nellie Kamkar, ${ }^{2,3}$ Jesper Ryg (D) ,,5,6 Tahir Masud, ${ }^{7}$ \\ Joost Daams, ${ }^{8}$ Manuel M Montero-Odasso, ${ }^{9,10,11}$ Sirpa Hartikainen, ${ }^{12}$ \\ Mirko Petrovic, ${ }^{13}$ Nathalie van der Velde, ${ }^{1}$ on behalf of the Task Force on Global \\ Guidelines for Falls in Older Adults
}

To cite: Seppala LJ, Kamkar N, Ryg J, et al. Protocol for a systematic review and meta-analysis assessing the effectiveness of deprescribing in falls prevention in older people. BMJ Open 2021;11:e047190. doi:10.1136/ bmjopen-2020-047190

- Prepublication history and additional supplemental material for this paper are available online. To view these files, please visit the journal online (http://dx.doi.org/10.1136/ bmjopen-2020-047190).

Received 24 November 2020 Accepted 21 September 2021

\section{D) Check for updates}

(c) Author(s) (or their employer(s)) 2021. Re-use permitted under CC BY-NC. No commercial re-use. See rights and permissions. Published by BMJ.

For numbered affiliations see end of article.

\section{Correspondence to} Dr Nathalie van der Velde; n.vandervelde@amsterdamumc. $\mathrm{nl}$

\section{ABSTRACT}

Introduction One of the known risk factors for fall incidents is the use of specific medications, fall-riskincreasing drugs (FRIDs). However, to date, there is uncertainty related to the effectiveness of deprescribing as a single intervention in falls prevention. Thus, a comprehensive update of the literature focusing on all settings in which older people receive healthcare and all deprescribing interventions is warranted to enhance the current knowledge.

Methods and analysis This systematic review protocol follows the Preferred Reporting Items for Systematic Reviews and Meta-Analyses guidelines. A systematic search was performed in Cochrane Central Register of Controlled Trials, MEDLINE, Embase and PsycINF0 (2 November 2020). We will also search in trial registers. We will include randomised controlled trials, in which any deprescribing intervention is compared with usual care and reports falls as an outcome. Both title and abstract screening and full-text screening will be done by two reviewers. The Cochrane Collaboration revised tool of Risk of Bias will be applied to perform risk of bias assessment. We will categorise the results separately for every setting. If a group of sufficiently comparable studies will be identified, we will perform a meta-analysis applying random effects model. We will investigate heterogeneity using a combination of visual inspection of the forest plot along with consideration of the $\chi^{2}$ test and the $\mathrm{I}^{2}$ statistic results. We have prespecified several subgroup and sensitivity analyses.

Ethics and dissemination Ethics approval is not applicable for this study since no original data will be collected. The results will be disseminated through peerreviewed publication and conference presentations. Furthermore, this systematic review will inform the recommendations of working group of polypharmacy and FRIDs of the anticipated World's Falls Guidelines. PROSPERO registration number CRD42020218231.

\section{BACKGROUND}

Fall incidents are a growing major public health concern leading to associated morbidity, mortality and substantial

\section{Strengths and limitations of this study}

- We aim to create the most comprehensive systematic review of the effectiveness of deprescribing as a single intervention in falls prevention to date by focusing on all settings in which older people receive healthcare and all deprescribing interventions.

- We will use the rigorous methodology in accordance with the Cochrane handbook and the results will be reported as stated by Preferred Reporting Items for Systematic Reviews and Meta-Analyses statement.

- The search algorithm was developed by an experienced librarian and customised to four large databases.

- No language restriction will be applied in the selection of the studies.

- The certainty of the evidence of this systematic review may be limited by the limited number of studies available and the possible low quality of the individual studies.

healthcare costs. ${ }^{1}$ Of the community-dwelling older adults aged 65 years and older, approximately a third will sustain a fall each year. ${ }^{1}$ In long-term care, residents are even at higher risk of falls; more than half of the residents will fall each year. ${ }^{2}$ One of the well-established risk factors for falls is the use of specific medications, so-called fall-risk-increasing drugs (FRIDs). ${ }^{3-5}$ The prevalence of FRID use in older people with a fall-related injury is high, ranging from $65 \%$ to $93 \% .{ }^{6}$ Medication review is a common component of the multifactorial falls prevention intervention and the Cochrane review by Hopewell et al concluded that multifactorial interventions may reduce the rate of falls compared with usual care or attention control. ${ }^{7}$ However, to date, there is uncertainty related to the 
effectiveness of deprescribing as a single intervention in falls prevention.

Few systematic reviews and meta-analyses have aimed to summarise the evidence-related to deprescribing as a single intervention. ${ }^{6-11}$ A comparison of the conclusions of these systematic reviews is difficult due to the variation in included trials in the different reviews. The trials performed in long-term care settings or hospitals were summarised by Cameron et al in $2018 .^{9}$ They concluded that general medication review may make little or no difference to the rate of falls or risk of falling in long term care facilities. In addition, they identified only one deprescribing intervention study that was performed in a hospital. Furthermore, the Cochrane review by Gillespie et al in 2012 assessing fall prevention approaches in community-dwelling older adults identified a total of five studies investigating medication withdrawal as a single intervention. ${ }^{8}$ Two of the five included studies found an effect of the intervention. Page et al found in 2016 in their meta-analysis that deprescribing led to fewer falls overall but did not significantly improve the risk of experiencing at least one fall. ${ }^{11}$ However, very heterogeneous trials were pooled together from placebocontrolled psychotropics withdrawal in primary care to education programme regarding appropriate medication use for physicians in nursing homes. Furthermore, Hart et al concluded in 2020 that reducing FRIDs use as a stand-alone intervention may not be effective. ${ }^{6}$ However, only studies performed in older adults presenting with a fall-related injury or a history of falls were included in the review. The most recent meta-analysis on this topic by Lee $e t$ al found no effect of FRIDs deprescribing on fall outcomes. ${ }^{10}$ However, all studies assessing medication reviews and management with a broader focus on reducing polypharmacy and potentially inappropriate prescribing were excluded.

Thus, a comprehensive update of the literature focusing on all deprescribing interventions including medication reviews with broader focus is warranted to enhance current knowledge as important deprescribing trials have been published in recent years. Therefore, our aim is to perform a systematic review concerning the effectiveness of deprescribing (eg, including general medication reviews or FRIDs deprescribing) as a single intervention in falls prevention performed in any setting in which older people receive healthcare. Furthermore, we aim to report the results separately for each setting and perform a meta-analysis if sufficiently comparable studies will be identified.

\section{METHODS}

This systematic review will be conducted and reported following the Preferred Reporting Items for Systematic Reviews and Meta-Analyses (PRISMA) guidelines.

\section{Eligibility criteria}

Type of studies

Only randomised controlled trials (RCTs), including quasi-randomised trials (eg, allocation by alternation), cluster-randomised trials and trials in which treatment allocations are inadequately concealed, will be included. We will include studies without language restriction.

\section{Types of participants}

Trials will be considered for inclusion if they included participants aged $\geq 60$ years or if the majority of participants are aged $>65$ years or the mean age is $>65$ years. We will include trials from all settings, for example, community, hospital ward, long-term care facilities.

\section{Type of interventions}

The intervention can be any deprescribing intervention. 'Deprescribing' has been described as 'the process of withdrawal of an (inappropriate) medication, supervised by a health care professional with the goal of managing polypharmacy and improving outcomes'. ${ }^{12}$ The interventions can be, for example, pharmacist-led medication reviews, physician-led interventions, prescriber education programmes, multidisciplinary interventions or clinical decision support systems. The intervention can target specific drug classes (eg, psychotropics) or general medication regimen (ie, comprehensive medication review). The intervention might target multiple medication issues in case of comprehensive medication review in addition to withdrawal such as polypharmacy, non-adherence, education and starting medications. If deprescribing intervention is a part of a multi-modal intervention (eg, including an exercise component in addition to deprescribing), the study will be excluded.

\section{Type of control}

The comparison intervention will be usual care (ie, no deprescribing or no change in usual activities of care).

\section{Type of outcomes}

We will include trials that report raw data or statistics related to falls outcomes. We will include any type of falls outcome: number of falls, number of fallers/non-fallers/ frequent fallers, fall rate per person-year, and time to first fall. Our secondary outcome is injurious falls (eg, fallrelated fractures, fall-related hospital admissions or fallrelated healthcare use).

\section{Information sources}

A systematic search was performed in Cochrane Central Register of Controlled Trials, MEDLINE, Embase and PsycINFO to search for literature published from onset until 2 November 2020 which will be updated to prior manuscript submission. A customised search strategy was conducted for each database. We will also search in trial registers. In the case that a relevant conference abstract is identified, we will contact the authors to obtain full-text article. Reference lists of included studies, reviews (eg, 
Cochrane reviews) and falls prevention guidelines will be reviewed to identify additional studies.

\section{Search strategy}

A search for Medline is provided as an example and is available in online supplemental appendix 1 .

The search terms used were:

1. Deprescription: inappropriate prescribing, medication errors, deprescriptions, drug prescriptions, drug utilisation, dose in combination with reduction, polypharmacy or medication in combination with risk, management or review, harmful medication, medication reconciliation, appropriate in combination with prescribing or medicine or medication, prescribing problem, overprescribing, under prescribing, withdrawal or discontinuation or problem or alternative or change in combination with medicine, medication or drug or FRID or polypharmacy, antidepressant or antipsychotic.

2. Falls or healthcare assessment: accidental falls, fall, fell, stumble, slip, trip, physical self-maintenance, ambulatory, healthcare outcome assessment.

3. Geriatric: geriatric assessment, frail, elderly, aged, middle aged, nursing homes, homes for the aged, ageing, older person, older patient, senior, elder, geriatric, frailty, postmenopausal women, community-dwelling, resident, old people, old client, old adult, older man, older woman.

4. (1) AND (2) AND (3).

5. Prescribing tools: for example, STOPP, 'Screening Tool of Older Person's Prescriptions'.

6. (4) OR (5.

7. RCT: randomised, randomly, double-blind, controlled trial, controlled clinical trial.

8. (6 AND (7).

The search was built by an experienced clinical librarian. We used 30 potentially relevant test articles to test and build the search. These articles were a priori identified using the function similar articles in PubMed and by reading references of the selected articles. These test articles included also articles that were identified from systematic reviews on deprescribing and included falls as a secondary outcome and not as a main interest.

\section{Data records and management}

First, title and abstract screening will be done independently by two reviewers using Rayyan, a web-based systematic review programme. In case of disagreement, a third reviewer will be consulted. Following the title and abstract screening, a full-text screening will be done using Rayyan by two independent reviewers. A third reviewer will be consulted in case of disagreement. Reasons for exclusion of studies will be collected during the full-text screening phase.

Two authors will independently extract data from each article using a structured data collection form. In case of disagreement, a third reviewer will be consulted. The following information will be collected: study design, country, setting, inclusion criteria, total number of participants and age (mean and SD), intervention type, control type, all fall-related outcomes, and how collected, adjustment of outcomes if applicable, follow-up duration, compliance to the intervention and if the trials have reported possible adverse effects related to the intervention or economic outcomes. If data to be extracted are missing, incomplete or unclear, inquiries will be sent to the authors.

\section{Effect measures}

We will report the treatment effects between the intervention and control group as a rate ratio ( $\mathrm{RaR})$, a risk tatio (RR) and/or a HR and accompanying 95\% Cls.

For rate of falls, we will use RaR as a treatment effect measure and the rate is the total number of falls per unit of person time that falls were monitored. We will use the unadjusted $\mathrm{RaR}$, unless the adjustment is performed due to clustering. Furthermore, if needed due to missing reporting, we will calculate $\mathrm{RaR}$ from appropriate raw data if possible. For dichotomous outcomes for example, fallers or frequent fallers, we will use RR as a treatment effect measure. We will use the unadjusted RR, unless the adjustment is performed due to clustering. Furthermore, if needed due to missing reporting or if OR is reported, we will calculate RR from the raw data if possible. For survival time-to-event data, we will use HR as a treatment effect measure. We will use the unadjusted HR, unless the adjustment is performed due to clustering.

Furthermore, we will adjust for clustering, if not already done in the published report using intra-cluster coefficient estimates and average cluster size.

\section{Risk of bias}

Two reviewers will assess the risk of bias independently by applying the Cochrane Collaboration revised tool of Risk of Bias (RoB 2.0) to all the included studies. In case of disagreement, a third reviewer will be consulted. The tool covers five domains: bias arising from randomisation process, bias due to deviations from intended interventions, bias due to missing outcome data, bias in measurement of outcome, bias in selection of the reported result. In addition, an additional domain is available for cluster randomised trials; bias arising from identification or recruitment of individual participants within clusters. Each domain has signalling questions aiming to elicit relevant information. Responses to these questions are fed into algorithms to score each domain either low risk of bias, some concerns or high risk of bias. The scores of each domain are further mapped into overall risk-of-biasjudgement including categories of low risk of bias, some concerns and high risk of bias.

\section{Data synthesis}

We will categorise the results separately for every setting (eg, community, hospital or long-term care facilities) due to the different participant and environment characteristics. 
First, a narrative synthesis will be provided in the text and tables to summarise the study characteristics and results.

If a group of studies with a sufficiently comparable intervention and outcome and performed in the same setting is identified, we will perform a meta-analysis applying the intention-to-treat principle. For example, a study purely investigating antihypertensive withdrawal will not be pooled with a study purely investigating antidepressant withdrawal.

The results will be pooled using a random-effects model considering the expected heterogeneity between the studies. We will try to minimise the heterogeneity by grouping the trials by setting and similar intervention. We will investigate remaining heterogeneity within a pooled group of trials using a combination of visual inspection of the forest plot along with consideration of the $\chi^{2}$ test (with statistical significance set at $\mathrm{p}<0.10$ ), and the $\mathrm{I}^{2}$ statistic results according to the recommendations from the Cochrane Handbook. We will explore heterogeneity by conducting a subgroup analysis based on the following: (a) age, (b) whether the trial is targeted to known fallers (or recurrent fallers if applicable) or also to non-fallers, (c) healthcare professionals conducting the medication review, for example, by physician or pharmacist, (d) whether the medication review is done with the help of a prescribing tool, for example, STOPP/START or the Beers criteria and which tool is used and (e) population, for example, if the trial is conducted only in dementia patients in comparison to general nursing home population. We will perform a sensitivity analysis according to overall study quality; low risk of bias, some concerns and high risk of bias, by comparing random and fixed-effect model and by excluding possible outlying studies, if the visual inspection of the forest plot shows poorly overlapping CIs.

We will explore the possibility of publication bias by constructing funnel plots and by conducting Egger's test for analyses that contain more than 10 studies.

The software Review Manager (RevMan) will be used for all statistical tests (Review Manager (RevMan) (Computer program), V.5.3, Copenhagen: The Nordic Cochrane Centre, The Cochrane Collaboration, 2014).

\section{Confidence in cumulative evidence}

The confidence in effect estimates for each reported outcome will be assessed using the Grading of Recommendations, Assessment, Development and Evaluation approach by two reviewers and possible disagreement will be assessed by third reviewer.

\section{Ethics and dissemination}

Ethics approval is not applicable for this study since no original data will be collected. The results will be disseminated through peer-reviewed publication and conference presentations. Furthermore, this systematic review will inform the recommendations of working group of polypharmacy and FRIDs of the anticipated World's Falls Guidelines.

\section{Patient and public involvement}

Patients or the public were not involved in the design, or conduct, or reporting, or dissemination plans of our research.

\section{DISCUSSION}

Although FRIDs use is an important risk factor for falls, there is uncertainty regarding the effectiveness of deprescribing interventions as a single intervention in falls prevention in older people. Identifying effective falls prevention interventions is of importance, considering the burden-related to fall injuries to both individuals and society.

This systematic review will help update the knowledge on the effectiveness of deprescribing, since we aim to create the most comprehensive systematic review to date by exploring all settings in which older people receive healthcare and all deprescribing interventions. In addition, we will use rigorous methodology in accordance with the Cochrane handbook and the results will be reported as stated by PRISMA statement. Therefore, we will provide relevant knowledge that will be implemented into anticipated World's Falls Guidelines and may influence future clinical practice. However, the certainty of the evidence of this systematic review may be limited by the limited number of studies available and the possible low quality of the individual studies.

\section{Author affiliations}

${ }^{1}$ Department of Internal Medicine, Section of Geriatric Medicine, Amsterdam Public Health Research Institute, Amsterdam UMC Location AMC, Amsterdam, The Netherlands

${ }^{2}$ Gait and Brain Laboratory, Lawson Research Health Institute, Parkwood Hospital, London, Ontario, Canada

${ }^{3}$ Department of Epidemiology and Biostatistics, University of Western Ontario, London, Ontario, Canada

${ }^{4}$ Department of Geriatric Medicine, Odense University Hospital, Odense, Denmark ${ }^{5}$ Geriatric Research Unit, Department of Clinical Research, University of Southern Denmark, Odense, Denmark

${ }^{6}$ ODIN (Odense Depresscibing INitiative), Odense, Denmark

${ }^{7}$ Nottingham University Hospitals NHS Trust, Nottingham, UK

${ }^{8}$ Research Support, Medical Library, Amsterdam UMC, University of Amsterdam, Amsterdam, The Netherlands

${ }^{9}$ Gait and Brain Lab, Lawson Research Health Institute, Parkwood Hospital, London, Ontario, Canada

${ }^{10}$ Departments of Medicine (Geriatrics) and of Epidemiology and Biostatistics, University of Western Ontario, London, Ontario, Canada

${ }^{11}$ Schulich School of Medicine and Dentistry, London, Ontario, Canada

${ }^{12}$ School of Pharmacy, University of Eastern Finland, Kuopio, Finland

${ }^{13}$ Department of Internal Medicine and Paediatrics (Section of Geriatrics), Ghent University, Ghent, Belgium

Collaborators Task Force on Global Guidelines for Falls in Older Adults:Mirko Petrovic, Alice Nieuwboer, Ellen Vlaeyen, Koen Milisen, Jesper Ryg, Rose Anne Kenny, Robert Bourke, Sirpa Hartikainen, Tischa Van der Cammen, Nathalie Van der Velde, Tahir Masud, Chris Todd, Finbarr C. Martin, David R. Marsh, Sallie Lamb, James Frith, Pip Logan, Dawn Skelton, Hubert Blain, Cedric Anweiller, Ellen Freiberger, Clemens Becker, Matteo Cesari, Alvaro Casas-Herrero, Javier Perez Jara, Christina Alonzo Bouzòn, Ana-Karim Welmer, Stephanie Birnghebuam, Reto 
Kressig, Manuel Montero-Odasso, Mark Speechley, Bill Mcllroy, Susan Hunter, Richard Camicioli, Kenneth Madden, Mireille Norris, Jennifer Watt, Louise Mallet, David B. Hogan, Joe Verghese, Ervin Sejdic, Luigi Ferruci, Lewis Lipsitz, David A. Ganz, Neil B. Alexander, Fabiana Giber, Marcelo Schapira, Ricardo Jauregui, Felipe Melgar-Cuellar, Roberto Alves Lourenço, Monica Pierrucini, Alejandro Ceriani, Pedro Marín-Larraín, Homero Gac Espinola, José Fernando Gómez-Montes, Carlos Alberto Cano-Gutierrez, Xinia Ramirez Ulate, José Ernesto Picado Ovares, Patricio Gabriel Buendia, Susana Lucia Tito, Diego Martínez Padilla, Sara G. Aguilar-Navarro, Alberto Mimenza, Rogelio Moctezum, Alberto Avila-Funes, Luis Miguel GutiérrezRobledo, Luis Manuel Cornejo Alemán, Edgar Aguilera Caona, Juan Carlos Carbajal, José F. Parodi, Aldo Sgaravatti, Stephen Lord,Cathie Sherrington, Cathy Said, lan Cameron, Meg Morris, Gustavo Duque, Jacqueline Close, Ngaire Kerse, Maw Pin Tan, Leilei Duan, Ryota Sakurai, Chek Hooi Wong, Chang Won Won, Jeffrey Hausdorff, Sebastiana Kalula, Olive Kobusingye. The steering committee consists following members: Clemens Becker, Hubert Blain, Jacqueline Close, Leilei Duan, David A. Ganz, David B. Hogan, Susan Hunter, Ricardo Jauregui, Rose Anne Kenny, Sallie Lamb, Lewis Lipsitz, Pip Logan, Stephen R. Lord, David R. Marsh, Finbarr C. Martin, Tahir Masud (co-chair), Koen Milisen, Manuel Montero-Odasso (chair), José Fernando Gómez-Montes, Mirko Petrovic, Jesper Ryg, Cathie Sherrington, Dawn Skelton, Maw Pin Tan, Nathalie van der Velde (co-chair), Joe Verghese, Nellie Kamkar (project manager).Steering Committee (worldfallsguidelines.com)

Contributors LJS and NvdV designed the protocol. LJS and NvdV wrote the first draft of the protocol. NK, JR, TM, JD, MMM-0, SH and MP provided critical appraisal regarding the design of the systematic review and revised the manuscript. JD designed and performed the search. All the authors approved the final version of the protocol.

Funding This work was supported by funding from the Canadian Institute of Health Research (CIHR; MOP 211220; PTJ 153100) and the Clementine Brigitta Maria Dalderup fund (grant number 7303), which is an Amsterdam University fund. The sponsors played no part in the design and writing of the protocol.

Competing interests None declared.

Patient consent for publication Not applicable.

Provenance and peer review Not commissioned; externally peer reviewed.

Supplemental material This content has been supplied by the author(s). It has not been vetted by BMJ Publishing Group Limited (BMJ) and may not have been peer-reviewed. Any opinions or recommendations discussed are solely those of the author(s) and are not endorsed by BMJ. BMJ disclaims all liability and responsibility arising from any reliance placed on the content. Where the content includes any translated material, BMJ does not warrant the accuracy and reliability of the translations (including but not limited to local regulations, clinical guidelines, terminology, drug names and drug dosages), and is not responsible for any error and/or omissions arising from translation and adaptation or otherwise.

Open access This is an open access article distributed in accordance with the Creative Commons Attribution Non Commercial (CC BY-NC 4.0) license, which permits others to distribute, remix, adapt, build upon this work non-commercially, and license their derivative works on different terms, provided the original work is properly cited, appropriate credit is given, any changes made indicated, and the use is non-commercial. See: http://creativecommons.org/licenses/by-nc/4.0/.

\section{ORCID iDs}

Lotta J Seppala http://orcid.org/0000-0001-9197-1424

Jesper Ryg http://orcid.org/0000-0002-8641-3062

\section{REFERENCES}

1 Berry SD, Miller RR. Falls: epidemiology, pathophysiology, and relationship to fracture. Curr Osteoporos Rep 2008;6:149-54.

2 Kennedy CC, loannidis G, Thabane L, et al. Successful knowledge translation intervention in long-term care: final results from the vitamin D and osteoporosis study (ViDOS) pilot cluster randomized controlled trial. Trials 2015;16:214.

3 de Vries M, Seppala LJ, Daams JG, et al. Fall-risk-increasing drugs: a systematic review and meta-analysis: I. cardiovascular drugs. J Am Med Dir Assoc 2018;19:371.e1-9.

4 Seppala LJ, van de Glind EMM, Daams JG, et al. Fall-risk-increasing drugs: a systematic review and meta-analysis: III. others. J Am Med Dir Assoc 2018;19:372.e1-8.

5 Seppala LJ, Wermelink AMAT, de Vries M, et al. Fall-risk-increasing drugs: a systematic review and meta-analysis: Il. psychotropics. $J$ Am Med Dir Assoc 2018;19:371.e11-371.e17.

6 Hart LA, Phelan EA, Yi JY, et al. Use of fall risk-increasing drugs around a fall-related injury in older adults: a systematic review. J Am Geriatr Soc 2020;68:1334-43.

7 Hopewell S, Adedire O, Copsey BJ, et al. Multifactorial and multiple component interventions for preventing falls in older people living in the community. Cochrane Database Syst Rev 2018;7:CD012221.

8 Gillespie LD, Robertson MC, Gillespie WJ, et al. Interventions for preventing falls in older people living in the community. Cochrane Database Syst Rev 2012;9:CD007146.

9 Cameron ID, Dyer SM, Panagoda CE, et al. Interventions for preventing falls in older people in care facilities and hospitals. Cochrane Database Syst Rev 2018;9:CD005465.

10 Lee J, Negm A, Peters R, et al. Deprescribing fall-risk increasing drugs (FRIDs) for the prevention of falls and fall-related complications: a systematic review and meta-analysis. BMJ Open 2021;11:e035978.

11 Page AT, Clifford RM, Potter K, et al. The feasibility and effect of deprescribing in older adults on mortality and health: a systematic review and meta-analysis. Br J Clin Pharmacol 2016;82:583-623.

12 Reeve E, Gnjidic D, Long J, et al. A systematic review of the emerging definition of 'deprescribing' with network analysis: implications for future research and clinical practice. $\mathrm{Br} J$ Clin Pharmacol 2015;80:1254-68. 